\title{
Constant Propagation on the Value Graph: Simple Constants and Beyond
}

\author{
Jens Knoop and Oliver Rüthing \\ Universität Dortmund, \\ Baroper Str. 301, D-44221 Dortmund, Germany \\ \{knoop, ruething\}@ls5.cs.uni-dortmund.de \\ http://sunshine.cs.uni-dortmund.de/
}

\begin{abstract}
We present a new algorithm for constant propagation $(C P)$, which works on the value graph of a program. This algorithm combines the (1) efficiency of the sparse CP-algorithms of Reif and Lewis [24,25], Ferrante and Ottenstein [9], and Wegman and Zadeck [30,31] with the (2) conceptual simplicity of their classic conventional counterparts of Kildall [19], and Kam and Ullman [16], while (3) outperforming them in power. It detects a proper superset of the simple constants detected by the classical $\mathrm{CP}$-algorithms, while avoiding the exponential worstcase behaviour of the CP-algorithm for finite constants of Steffen and Knoop [28] allowing its optimality for acyclic control flow. The class detected is a well-balanced compromise between power and performance. In fact, we show that $\mathrm{CP}$ is co-NP-hard for acyclic control flow making the existence of efficient optimal algorithms for acyclic control flow most unlikely. Nonetheless, the new algorithm is open for extensions, e.g. towards conditional constants in the fashion of $[30,31]$ in order to capture to a certain extent the determinism of conditional branching.
\end{abstract}

\section{Motivation}

Constant propagation $(C P)$ is one of the most widely used optimisations in practice (cf. [1,18,23,32]). Intuitively, it aims at replacing terms which always yield a unique constant value at run-time by this value as illustrated in Figure 1.

Pioneering for CP has been the algorithm of Kildall [19], which detects the class of so-called simple constants. Intuitively, a term is a simple constant, if it is a constant, or if all its operands are simple constants. Though simple constants fail optimality even for acyclic programs, i.e., there are terms being constant without being a simple constant, the class of simple constants is still dominating in practice and computed by state-of-the-art optimising compilers. This may have two reasons. First, the general problem of detecting constancy of terms is undecidable (cf. $[24,25]$ ); second, decidable supersets of simple constants, which have been proposed in the literature are usually based on simple heuristics of quite limited power as the one of Kam and Ullman [16], or are accompanied by computationally expensive algorithms like the one of Steffen and Knoop [28] deciding the class of finite constants. In the worst case, this algorithm is exponential in the program size, but unique in being optimal for acyclic programs. 
a)

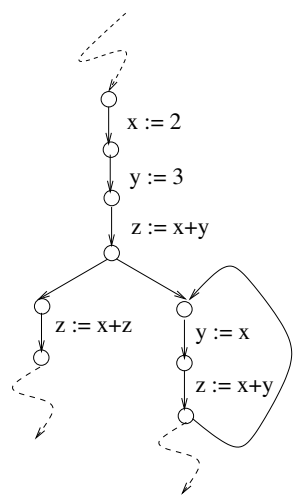

Original program b)

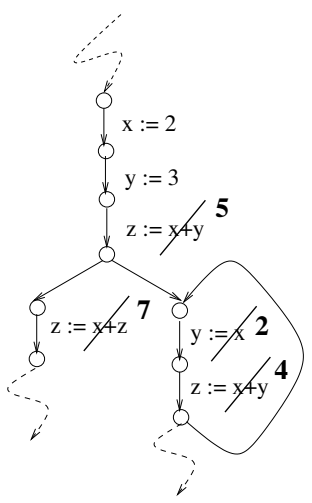

After simple constant propagation

Fig. 1. Illustrating (simple) constant propagation.

The research on CP can actually roughly be split into two major groups. First, approaches aiming at making CP available for more general program settings like the interprocedural (cf. $[3,8,12,22,27]$ ) or (explicitly) parallel one (cf. $[20,21]$ ), which (usually) focus on proper subsets of simple constants like copy constants or linear constants. Second, approaches (cf. [9,24,25,30,31]) ${ }^{1}$ aiming at improving the efficiency of the classical algorithms for simple constants of Kildall [19], and Kam and Ullman [16].

Fundamental for the success of the algorithms of the second group, which are known as sparse CP-algorithms, is the use of specific representations of control and data-flow information like the global value graph $[24,25],{ }^{2}$ the program dependence graph [9], or the $S S A$ form of a program [30,31].

The approach we propose here starts from the line of these algorithms. It performs $\mathrm{CP}$ on the value graph of a program [2]. ${ }^{2}$ The value graph represents uniformly and concisely the control and the data flow of a program, and has originally been designed and used so far as a means for efficiently detecting equivalent program terms $[2,26]$.

In this article, we demonstrate that it is well-suited for CP, too. In fact, the new algorithm combines the (1) efficiency of the sparse algorithms of Reif and Lewis [24,25], Ferrante and Ottenstein [9], and Wegman and Zadeck [30,31] with the (2) conceptual simplicity of their classic conventional counterparts of Kildall [19], and Kam and Ullman [16], while (3) outperforming them in power. It detects a proper superset of the simple constants detected by its classical

\footnotetext{
${ }^{1}$ Wegman and Zadeck start from a basic algorithm capturing simple constants, while actually aiming at an algorithm capturing a more general class called conditional constants, which capture some aspects of conditional program branching.

2 The global value graph introduced by Reif and Lewis should not be confused with the value graph introduced by Alpern, Wegman, and Zadeck [2] underlying our approach. These are different data structures (see also Section 5.4).
} 
counterparts as illustrated in Figure 2, while avoiding the exponential worstcase behaviour of the CP-algorithm for finite constants of Steffen and Knoop [28] allowing the optimality of their algorithm for acyclic flow.

a)

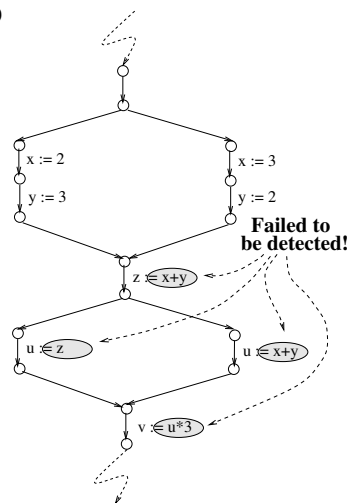

After simple constant propagation (Note: No effect at all!) b)

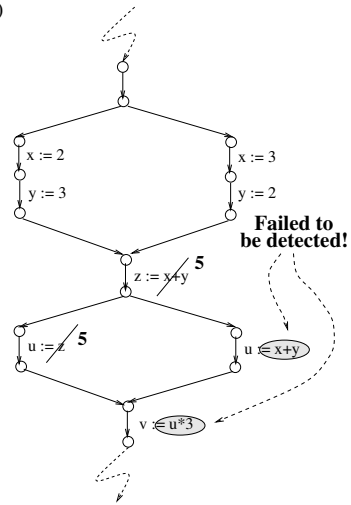

After simple constant propagation enriched by the "look-ahead-of-one" heuristics of Kam and Ullman c)

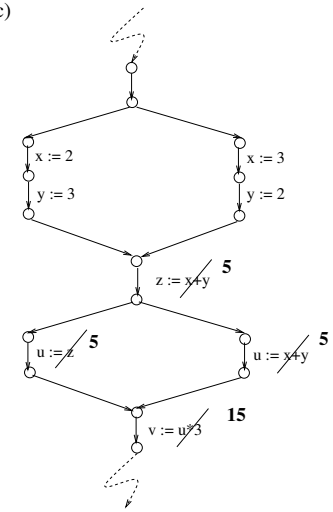

The effect of the new algorithm

Fig. 2. Motivating example.

Moreover, the new algorithm, which works for arbitrary control flow, is scalable. While its basic version detects the class of simple constants, the class detected by the full algorithm goes well-beyond. It is a well-balanced compromise between power and performance. In fact, we show that $\mathrm{CP}$ is co-NP-hard for acyclic control flow. This makes the existence of efficient optimal algorithms for acyclic control flow most unlikely. Nonetheless, the new algorithm is open for extensions, e.g. towards conditional constants in the fashion of [30,31] in order to capture to some extent the determinism of conditional branching.

\section{Preliminaries}

As usual we represent programs by directed flow graphs $G=(N, E, \mathbf{s}, \mathbf{e})$ with node set $N$, edge set $E$, a unique start node $\mathbf{s}$, and a unique end node $\mathbf{e}$, which are assumed to have no predecessors and successors, respectively. Edges represent the branching structure and the statements of a program, while nodes represent program points. Unlabelled edges are assumed to represent "skip."

By $\operatorname{pred}(\mathbf{n})=_{d f}\{\mathbf{m} \mid(\mathbf{m}, \mathbf{n}) \in E\}$ and $\operatorname{succ}(\mathbf{n})=_{d f}\{\mathbf{m} \mid(\mathbf{n}, \mathbf{m}) \in E\}$ we denote the set of immediate predecessors and successors of a node $\mathbf{n}$. Additionally, by source $(e)$ and $d s t(e), e \in E$, we denote the source node and the destination node of edge $e$. A finite path in $G$ is a sequence $\left(e_{1}, \ldots, e_{q}\right)$ of edges such that $\operatorname{dst}\left(e_{j}\right)=\operatorname{source}\left(e_{j+1}\right)$ for $j \in\{1, \ldots, q-1\}$. It is called a path from $\mathbf{m}$ to $\mathbf{n}$, if $\operatorname{source}\left(e_{1}\right)=\mathbf{m}$ and $d s t\left(e_{q}\right)=\mathbf{n}$. By $\mathbf{P}[\mathbf{m}, \mathbf{n}]$ we denote the set of all finite paths 
from $\mathbf{m}$ to $\mathbf{n}$. Without loss of generality we assume that every node of a flow graph $G$ lies on a path from $\mathbf{s}$ to e.

\section{Simple Constants: The Conventional Approach}

In this section we recall the essence of the classical algorithms of Kildall [19], and of Kam and Ullman [16] for simple constants. Simultaneously, this establishes the basics for our new value-graph based CP-algorithm.

Semantics of Terms. Let terms $t \in \mathbf{T}$ be inductively built from variables $v \in \mathbf{V}$, constants $c \in \mathbf{C}$, and operators op $\in \mathbf{O p}$ of arity $r \geq 1 .^{3}$ The semantics of terms is then induced by an interpretation $I=\left(\mathbf{D}^{\prime} \cup\{\perp, \top\}, I_{0}\right)$, where $\mathbf{D}^{\prime}$ denotes a non-empty data domain, $\perp$ and $\top$ two new data not in $\mathbf{D}^{\prime}$, and $I_{0}$ a function mapping every constant $c \in \mathbf{C}$ to a datum $I_{0}(c) \in \mathbf{D}^{\prime}$, and every $r$ ary operator $o p \in \mathbf{O p}$ to a total function $I_{0}(o p): \mathbf{D}^{r} \rightarrow \mathbf{D}, \mathbf{D}={ }_{d f} \mathbf{D}^{\prime} \cup\{\perp, \top\}$ being strict in $\perp$ and $\top$ with $\perp$ prioritised over $\top$ (i.e., $I_{0}(o p)\left(d_{1}, \ldots, d_{r}\right)=\perp$, whenever there is a $j, 1 \leq j \leq r$, with $d_{j}=\perp$, and $I_{0}(o p)\left(d_{1}, \ldots, d_{r}\right)=\top$, whenever there is no $j, 1 \leq j \leq r$, with $d_{j}=\perp$, but a $j, 1 \leq j \leq r$, with $d_{j}=\top$ ). $\Sigma={ }_{d f}\{\sigma \mid \sigma: \mathbf{V} \rightarrow \mathbf{D}\}$ denotes the set of states, and $\sigma_{\perp}$ the distinct start state assigning $\perp$ to all variables $v \in \mathbf{V}$. This choice reflects that we do not assume anything about the context of a program being optimised. The semantics of a term $t \in \mathbf{T}$ is then given by the inductively defined evaluation function $\mathcal{E}: \mathbf{T} \rightarrow(\Sigma \rightarrow \mathbf{D}):$

$\forall t \in \mathbf{T} \forall \sigma \in \Sigma \cdot \mathcal{E}(t)(\sigma)={ }_{d f} \begin{cases}\sigma(x) & \text { if } t=x \in \mathbf{V} \\ I_{0}(c) & \text { if } t=c \in \mathbf{C} \\ I_{0}(o p)\left(\mathcal{E}\left(t_{1}\right)(\sigma), \ldots, \mathcal{E}\left(t_{r}\right)(\sigma)\right) & \text { if } t=o p\left(t_{1}, \ldots, t_{r}\right)\end{cases}$

For convenience we assume $\mathbf{D}^{\prime} \subseteq \mathbf{T}$, i.e., we identify the set of data $\mathbf{D}^{\prime}$ with the set of constants C. By means of the state transformation function $\theta_{\iota}: \Sigma \rightarrow \Sigma$, $\iota \equiv x:=t$, which is defined by

$$
\forall \sigma \in \Sigma \forall y \in \mathbf{V} . \theta_{\iota}(\sigma)(y)={ }_{d f} \begin{cases}\mathcal{E}(t)(\sigma) & \text { if } y=x \\ \sigma(y) & \text { otherwise }\end{cases}
$$

we obtain the set of states $\Sigma_{\mathbf{n}}={ }_{d f}\left\{\theta_{p}\left(\sigma_{\perp}\right) \mid p \in \mathbf{P}[\mathbf{s}, \mathbf{n}]\right\}$, which are possible at a program point $\mathbf{n} \in N .{ }^{4}$ By means of $\Sigma_{\mathbf{n}}$, we can now define the set $\mathcal{C}_{\mathbf{n}}$ of all terms yielding at run-time a unique constant value at a program point $\mathbf{n} \in N$ :

$$
\mathcal{C}_{\mathbf{n}}={ }_{d f}\left\{(t, d) \in \mathbf{T} \times \mathbf{D}^{\prime} \mid \forall \sigma \in \Sigma_{\mathbf{n}} \cdot \mathcal{E}(t)(\sigma)=d\right\}
$$

Unfortunately, the sets $\mathcal{C}_{\mathbf{n}}, \mathbf{n} \in N$, are generally not decidable (cf. [24]). Simple constants recalled next are an efficiently decidable subset of $\mathcal{C}_{\mathbf{n}}$, which are computed by state-of-the-art optimisers.

\footnotetext{
${ }^{3}$ Note, during the development of our algorithm we will consider terms with at most one operator, and assume that all operators are binary.

${ }^{4}$ In the definition of $\Sigma_{\mathbf{n}}, \theta_{p}$ denotes the straightforward extension of the state transformation functions to paths.
} 
Simple Constants. Intuitively, a term is a simple constant (SC) (cf. [19]), if it is a constant or if all its operands are simple constants. For example, all righthand side terms in the example of Figure 1(a) are simple constants, while those (except for the right-hand side terms 2 and 3) in the example of Figure 2(a) are not. The classical algorithms of Kildall, and Kam and Ullman rely on data-flow analysis for computing SCs. Its essence is recalled in the following section.

Data-Flow Analysis. In essence, data-flow analysis (DFA) aims at computing information about the program states, which may occur at specific program points at run-time. Theoretically well-founded are DFAs based on abstract interpretation (cf. [6]). An abstract semantics is usually specified by a local semantic functional $\llbracket \rrbracket: E \rightarrow(\mathcal{C} \rightarrow \mathcal{C})$ giving abstract meaning to every program statement, i.e., to every edge $e \in E$ of a flow graph $G$, in terms of a transformation function on a complete lattice $(\mathcal{C}, \sqcap, \sqsubseteq, \perp, \top)$. Its elements express the DFAinformation of interest.

Local semantic functionals can easily be extended to capture finite paths, which provides the key to the meet over all paths (MOP) approach in the sense of Kam and Ullman [16]. It yields the intuitively desired solution of a DFA-problem by directly mimicking possible program executions: it "meets" all informations belonging to a program path leading from $\mathbf{s}$ to a program point $\mathbf{n} \in N$.

$$
\text { The MOP-Solution: } \forall c_{0} \in \mathcal{C} . \operatorname{MOP}(\mathbf{n})\left(c_{0}\right)=\prod\left\{\llbracket p \rrbracket\left(c_{0}\right) \mid p \in \mathbf{P}[\mathbf{s}, \mathbf{n}]\right\}
$$

Unfortunately, this is in general not effective. The $M O P$-approach is thus complemented by the maximal fixed point (MFP) approach in the sense of Kam and Ullman [16]. Intuitively, it approximates the greatest solution of a system of equations imposing consistency constraints on an annotation of the program with DFA-information with respect to some start information $c_{0} \in \mathcal{C}$ :

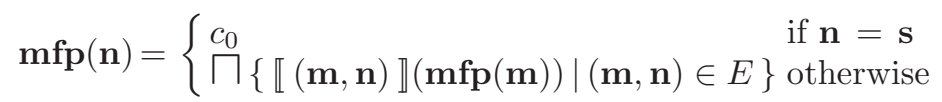

The greatest solution of this equation system, which we denote by $\mathrm{mf}_{c_{0}}$, can effectively be computed, if the semantic functions $\llbracket e \rrbracket, e \in E$, are monotonic, and the lattice $\mathcal{C}$ satisfies the descending chain condition. It defines the solution of the $M F P$-approach, which is usually considered the minimal acceptable criterion of the quality of a data-flow analysis (cf. $[11,16])$ :

The MFP-Solution: $\forall c_{0} \in \mathcal{C} \forall \mathbf{n} \in N . \operatorname{MFP}(\mathbf{n})\left(c_{0}\right)={ }_{d f} \operatorname{mfp}_{c_{0}}(\mathbf{n})$

The following theorem, which relates both solutions, is central. It gives sufficient conditions for the safety (correctness) and precision (optimality) of the MFPsolution with respect to the $M O P$-solution (cf. $[16,19])$ :

\section{Theorem 1 (Safety and Coincidence Theorem).}

1. Safety: $\operatorname{MFP}(\mathbf{n}) \sqsubseteq M O P(\mathbf{n})$, if all $\llbracket e \rrbracket, e \in E$, are monotonic.

2. Coincidence: $\operatorname{MFP}(\mathbf{n})=\operatorname{MOP}(\mathbf{n})$, if all $\llbracket e \rrbracket, e \in E$, are distributive. 
Computing Simple Constants. Considering D a flat lattice, the set of states $\Sigma$ together with the pointwise ordering is a complete lattice, too. Having this in mind, the computation of SCs relies on the local semantic functional $\llbracket \rrbracket_{s c}$ : $E \rightarrow(\Sigma \rightarrow \Sigma)$ defined by $\llbracket e \rrbracket_{s c}={ }_{d f} \theta_{e}$ for each $e \in E$, and the start state $\sigma_{\perp}$. Since the number of variables in a program is finite, we have (cf. [16]):

Lemma 1. $\Sigma$ is of finite height, and all functions $\llbracket e \rrbracket_{s c}, e \in E$, are monotonic.

In general, the functions $\llbracket e \rrbracket_{s c}, e \in E$, are not distributive (cf. [16]). However, as pointed out before, monotonicity together with the finite height of the data-flow lattice is sufficient for the effective computability of the MFP-solution of the SC-problem, which we denote by $M F P_{\sigma_{\perp}}^{s c} \cdot{ }^{5}$ It induces the formal definition of simple constants: $\mathcal{C}_{\mathbf{n}}^{s c}={ }_{d f} \mathcal{C}_{\mathbf{n}}^{s c, m f p}={ }_{d f}\left\{(t, d) \in \mathbf{T} \times \mathbf{D}^{\prime} \mid \mathcal{E}(t)\left(M_{F P} P_{\sigma_{\perp}}^{s c}(\mathbf{n})\right)=d\right\}$.

Defining dually the set of constants $\mathcal{C}_{\mathbf{n}}^{s c, m o p}={ }_{d f}\left\{(t, d) \in \mathbf{T} \times \mathbf{D}^{\prime} \mid \mathcal{E}(t)\left(M O P_{\sigma}^{s c}\right.\right.$ $(\mathbf{n}))=d\}$ induced by the $M O P_{\sigma_{\perp}}^{s c}$-solution, we obtain by means of Theorem $1(1)$ (cf. [16]):

Theorem 2 (SC-Correctness). $\forall \mathbf{n} \in N . \mathcal{C}_{\mathbf{n}} \supseteq \mathcal{C}_{\mathbf{n}}^{s c, m o p} \supseteq \mathcal{C}_{\mathbf{n}}^{s c, m f p}=\mathcal{C}_{\mathbf{n}}^{s c}$.

The algorithms of Kildall, and Kam and Ullman are both instances of the MFPapproach recalled above. While Kam and Ullman present their algorithm using environments in the sense of states introduced above, Kildall uses structured partitions for this purpose which results in a higher complexity of his algorithm.

\section{Constant Propagation on the Value Graph}

In this section we present the basic version of the new algorithm for $\mathrm{CP}$ working on the value graph. We thus recall first its definition.

\subsection{The Value Graph of Alpern, Wegman and Zadeck}

The value graph of Alpern, Wegman and Zadeck is a data structure, which uniformly and concisely combines the control and the data flow of a program [2]. It is constructed on top of the static single assignment ( $S S A$ ) form of a program (cf. [7]), which we illustrate here on an informal and intuitive level considering the program of Figure 3(a) as example.

In essence, in the SSA form of a program the variables of the original program are replaced by new versions such that every variable has a unique initialization point. At join points of the control flow pseudo-assignments $x_{k}:=$ $\phi_{\mathbf{n}}\left(x_{i 1}, \ldots, x_{i k}\right)$ are introduced meaning that $x_{k}$ gets the value of $x_{i j}$ if the join node is entered via the $j$ th ingoing edge. ${ }^{6}$ The SSA form of our running example is shown in Figure 3(b).

Based upon the SSA form of a program the value graph is constructed. It represents the value transfer of SSA variables along the control flow of the program. Following the description in [23] the value graph is defined as a labelled directed graph where

\footnotetext{
${ }^{5}$ Note that a lattice of finite height trivially satisfies the descending chain condition.

${ }^{6} \phi$-operators are indexed by their corresponding join node.
} 
a)

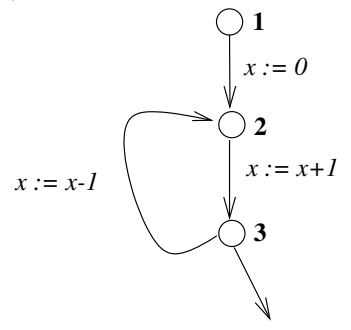

b)

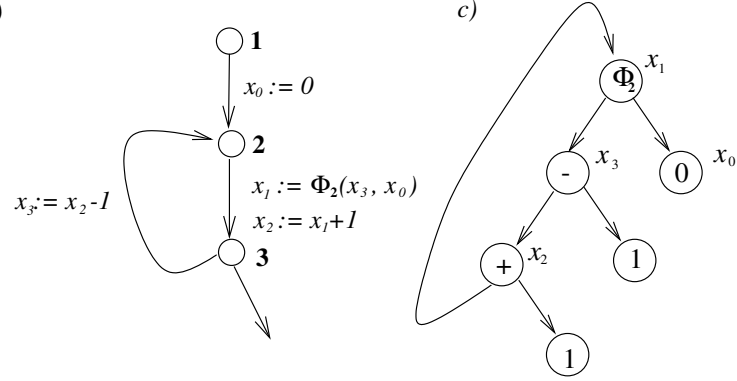

Fig. 3. The construction of the value graph: (a) the original program with $x$ being a simple constant of value 1 on the exit of the loop, (b) the program transformed into SSA form and (c) the corresponding value graph.

- the nodes correspond to occurrences of nontrivial assignments, i.e., assignments whose right-hand sides contain at least one operator, and to occurrences of constants in the program. ${ }^{7}$ Every node is labelled with the corresponding constant or operator symbol, and additionally with the set of variables whose value is generated by the corresponding constant or assignment. An operator node is always annotated with the left-hand side variable of its corresponding assignment. Note, the generating assignment of $x$ of a trivial assignment $x:=y$ is defined as the generating assignment of $y$. For a trivial assignment $x:=c$ variable $x$ is attached to the annotation of the corresponding node associated with $c$. For convenience, the constant or operator label is written inside the circle visualizing the node, and the variable annotation outside.

- Directed edges point to the operands of the right-hand side expression associated with the node. Moreover, edges are labelled with natural numbers according to the position of operands. ${ }^{8}$

Figure 3(c) shows the value graph corresponding to Figure 3(b). It is worth noting that it is cyclic which is due to self-dependencies of variables in the loop.

In the following we assume without loss of generality that all operators, i.e., ordinary ones as well as $\phi$-operators, are binary. ${ }^{9}$ In fact, this assumption is not crucial for our reasoning, but simplifies the formal development. It can easily be extended to capture $k$-ary operators as well. Moreover, for a node $n$ in the value graph we denote its operator or constant label by lab $[n] .{ }^{10}$ If it is an inner node, its left and right child nodes are denoted by $\mathrm{l}(n)$ and $\mathrm{r}(n)$, respectively.

$\overline{7}$ For the sake of simplicity it is assumed that variables are initialized.

${ }^{8}$ We omit this labelling in our examples making the implicit assumption that edges are ordered from left to right.

${ }^{9}$ For $\phi$-operators this assumption can always be achieved by "linearizing" join nodes having more than two incoming edges.

${ }^{10}$ For clarity, we denote flow graph nodes by boldface letters and value graph nodes by italic ones. 


\subsection{Computing Simple Constants on the Value Graph}

Simple constants can be computed very efficiently on the value graph, as propagtion of information is directly reflected in this structure. Essentially, this resembles to Wegman and Zadeck's sparse CP-algorithm on SSA-form [31]. However, the value graph allows a much simpler conceptual presentation as the problem reduces to a data-flow evaluation on the value graph. In details, the analysis on the value graph computes the greatest fixed point of a data-flow annotation dfi[.] by the following iterative procedure:

Start Annotation: For each node $n$ in the value graph initialize:

$$
\operatorname{dfi}[n]= \begin{cases}I_{0}(c) & \text { if } n \text { is a leaf node with } c={ }_{d f} \operatorname{lab}[n] \\ \top & \text { otherwise }\end{cases}
$$

\section{Iteration Step:}

1. For a node $n$ labelled with an ordinary operator $\omega$ :

$$
\operatorname{dfi}[n]=I_{0}(\omega)(\operatorname{dfi}[1(n)], \operatorname{dfi}[\mathrm{r}(n)]) \quad \text { (Evaluating terms) }
$$

2. For a node $n$ labelled with a $\phi$-operator $\phi_{\mathbf{n}}$ :

$$
\operatorname{dfi}[n]=\operatorname{dfi}[1(n)] \sqcap \operatorname{dfi}[\mathrm{r}(n)] \quad \text { (Merging DFA-info's at join nodes) }
$$

Figure 4 illustrates the iteration for the value graph of Figure 3(c). Note, in contrast to the classical algorithm of Kam and Ulman [16], which works on flow graphs storing a complete environment as data-flow information at every flow graph node, our algorithm works on the value graph storing a single data at every value graph node.

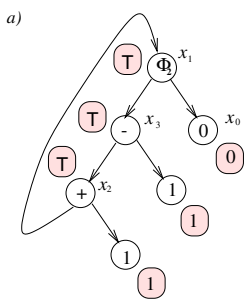

After the initialization step

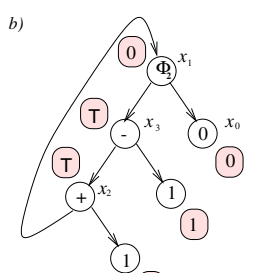

(1)

After the 1st iteration step

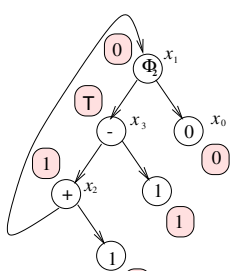

(1)

After the 2nd iteration step

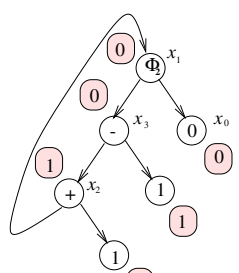

(1)

After the 3rd iteration step. Stable!

Fig. 4. The data-flow analysis on the value graph of Figure 3(c): (a) the start annotation and (b) the greatest fixed point annotation.

The time complexity of the algorithm is linear in the size of the value graph which, in the worst case, is of order $\mathcal{O}(|N||\mathbf{V}|)$. In practice, however, it usually behaves much better, and is expected to be linear [23]. Moreover, we have: 


\section{Theorem 3 (Basic Algorithm).}

The basic version of the new VG-based algorithm for CP detects the class of simple constants.

In the following section we show how to enhance this algorithm in order to detect proper supersets of the class of simple constants almost without losing efficiency.

\section{Beyond Simple Constants}

The value graph provides an elegant handle to design more powerful, yet still efficient algorithms that are capable of detecting much broader classes of constants than the class of simple ones. This concern has already been addressed in the past. However, state-of-the-art approaches are either limited to a few ad-hoc effects, like the "look-ahead-of-one" heuristics of Kam and Ullman [16], ${ }^{11}$ or are expensive in terms of computational complexity as e.g. the ambitious approach of Steffen and Knoop [28] computing the set of finite constants. As the most significant and distinguishing characteristics their algorithm is unique to detect in acyclic programs all constants. This, however, comes at the price of an exponential worst-case time complexity. In the light of the following contribution of this article, however, this is not too surprising. We demonstrate that the problem is intractable in general. Thereafter, we propose an algorithm which captures a reasonable and large subclass of finite constants which can efficiently be detected, and generalises on previous approaches going beyond simple constants, in particular the one of Kam and Ullman.

\subsection{Constant Propagation for Acyclic Control Flow is Co-NP-Hard}

We give a polynomial time reduction from 3-SAT, the satisfiability problem for clauses with three disjuncts (cf. [10]). To this end let $V=\left\{v_{1}, \ldots, v_{k}\right\}$ be a set of Boolean variables. A clause of size $s$ over $V$ is a disjunction of $s$ variables or negated variables in $V$. A truth assignment is a function $t: V \rightarrow \mathbb{B}$, where $\mathbb{B}$ denotes the set of Boolean truth values. Then the 3-SAT problem is as follows:

\section{3-SAT}

Instance: A set of clauses $C=\left\{c_{1}, \ldots, c_{n}\right\}$ over $V$ all being of size 3 .

Question: Is there a truth assignment $t$ such that all clauses in $C$ are satisfied?

3-SAT is known as an NP-complete problem (cf. [10]). In the following we give a polynomial time reduction of its co-problem, i.e., the question whether at least one clause is not satisfied by any truth assignment, to the constant propagation problem on acyclic flow graphs. We illustrate the reduction by means of a 3-SAT instance over the variables $\left\{v_{1}, \ldots, v_{k}\right\}$ given by :

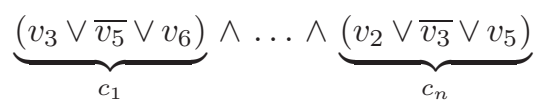

${ }^{11}$ In essence, this heuristics delays computing the meet of DFA-informations at join nodes of the control flow across them which results in a look-ahead of one statement (or basic block). The limitations of this heuristics are illustrated in Figure 2(b). 
This Boolean expression is coded into the flow graph depicted in Figure 5, where integer program variables $x_{i}$ and $\overline{x_{i}}$ are introduced for every Boolean variable $v_{i}$. Now, it is easy to see that $r 2$ is a constant of value 1 at the exit of the program fragment if and only if 3-SAT is not satisfiable. ${ }^{12}$ In fact, this follows from the following facts: $r 1$ is different from 0 on any program path if and only if the underlying instance of 3 -SAT is not satisfiable. ${ }^{13}$ Since in this case $r 1$ is in the range $\{1, \ldots, k\}$, the resulting variable $r 2$ is assigned to 1 .

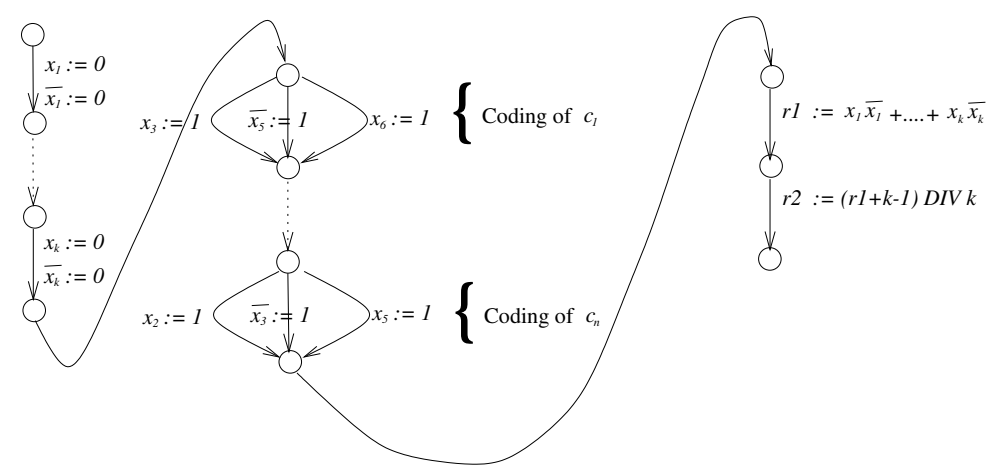

(I) Initializiation $\quad$ (II) Coding of Clauses

(III) "Non-Satisfiability Reduction"

Fig. 5. The co-NP-hardness reduction: $r 2$ evaluates to 1 if and only if the underlying 3-SAT instance is not satisfiable.

Hence, the existence of efficient algorithms, which are complete for acyclic control flow, is most unlikely. In the following section we thus present an algorithm efficiently determining a major fragment of the set of finite constants.

\subsection{An Efficiently Decidable Subset of Finite Constants}

Basically, our extension to the algorithm for simple constants refines the domain the local semantic functions are operating on. Instead of using data elements of $\mathbf{D}^{\prime}$ for the analysis we introduce $\phi$-constants over $\mathbf{D}^{\prime}$. These are expressions composed of constants and $\phi$-operators. Formally, $\mathbf{D}^{\phi}$ is defined inductively as the smallest set satisfying:

1. $\mathbf{D}^{\prime} \subseteq \mathbf{D}^{\phi}$

2. If $\phi_{\mathbf{n}}$ is a $\phi$-operator occurring in the SSA program and $d_{1}, d_{2} \in \mathbf{D}^{\phi}$ such that neither $d_{1}$ nor $d_{2}$ contains $\phi_{\mathbf{n}}$, then $\phi_{\mathbf{n}}\left(d_{1}, d_{2}\right) \in \mathbf{D}^{\phi}$.

$\overline{12 \text { Note that }+}$ and . denote addition and multiplication in the program.

13 Note that a truth assignment that satisfies all clauses would yield a path where for every $i \in\{1, \ldots, k\}$ not both $x_{i}$ and $\overline{x_{i}}$ are set to 1 . 
Adding a bottom and a top element to $\mathbf{D}^{\phi}$ makes $\mathbf{D}^{\phi} \cup\{\perp, \top\}$ a complete partial order $^{14}$ that relates $\phi$-constants with other elements in the following way:

$$
\phi_{\mathbf{n}}\left(r_{1}, r_{2}\right) \sqsubseteq r \Longleftrightarrow{ }_{d f}\left(r_{1} \sqsubseteq r \vee r_{2} \sqsubseteq r\right) \vee\left(r=\phi_{\mathbf{n}}\left(r_{3}, r_{4}\right) \wedge r_{1} \sqsubseteq r_{3} \wedge r_{2} \sqsubseteq r_{4}\right)
$$

If $r_{1}$ and $r_{2}$ are $\phi$-constants and $\omega \in \mathbf{O p}$ then $\omega\left(r_{1}, r_{2}\right)$ will be called an evaluation candidate. This is because such expressions are constructed during the analysis where they are immediately evaluated to data in $\mathbf{D}^{\phi} \cup\{\perp, \top\}$.

The Analysis. The analysis on the value graph works essentially by annotating nodes of the value graph by elements of the new domain $\mathbf{D}^{\phi} \cup\{\perp, \top\}$. The essential difference to the setting for the detection of simple constants is that $\phi$-nodes are now possibly evaluated by constructing $\phi$-constants whenever both operands are different $\phi$-constants. $\phi$-constants are then used to gain precision by evaluating ordinary operators in a way which is sensitive to $\phi$-operands. The evaluation function $\mathcal{E}^{+}$maps evaluation candidates to data in $\mathbf{D}^{\phi} \cup\{\perp, \top\}$.

1. $\mathcal{E}^{+}(d)=d$ if $d \in \mathbf{D}$

2. $\mathcal{E}^{+}\left(\phi_{\mathbf{n}}\left(r_{1}, r_{2}\right)\right)= \begin{cases}\perp & \text { if } \mathcal{E}^{+}\left(r_{1}\right) \text { or } \mathcal{E}^{+}\left(r_{2}\right) \text { contains } \phi_{\mathbf{n}} \\ \mathcal{E}^{+}\left(r_{1}\right) & \text { if } \mathcal{E}^{+}\left(r_{1}\right) \sqsubseteq \mathcal{E}^{+}\left(r_{2}\right) \\ \mathcal{E}^{+}\left(r_{2}\right) & \text { if } \mathcal{E}^{+}\left(r_{2}\right) \sqsubseteq \mathcal{E}^{+}\left(r_{1}\right) \\ \phi_{\mathbf{n}}\left(\mathcal{E}^{+}\left(r_{1}\right), \mathcal{E}^{+}\left(r_{2}\right)\right) & \text { otherwise }\end{cases}$

3. $\mathcal{E}^{+}\left(\omega\left(r_{1}, r_{2}\right)\right)=$

$$
\begin{cases}I_{0}(\omega)\left(r_{1}, r_{2}\right) & \text { if } r_{1}, r_{2} \in \mathbf{D} \\ \mathcal{E}^{+}\left(\phi_{\mathbf{n}}\left(\omega\left(r_{1}, r_{21}\right), \omega\left(r_{1}, r_{22}\right)\right)\right) & \text { if } r_{1} \in \mathbf{D}, r_{2}=\phi_{\mathbf{n}}\left(r_{21}, r_{22}\right) \\ \mathcal{E}^{+}\left(\phi_{\mathbf{n}}\left(\omega\left(r_{11}, r_{2}\right), \omega\left(r_{12}, r_{2}\right)\right)\right) & \text { if } r_{1}=\phi_{\mathbf{n}}\left(r_{11}, r_{12}\right), r_{2} \in \mathbf{D} \\ \mathcal{E}^{+}\left(\phi_{\mathbf{n}}\left(\omega\left(r_{11}, r_{21}\right), \omega\left(r_{12}, r_{22}\right)\right)\right) & \text { if } r_{1}=\phi_{\mathbf{n}}\left(r_{11}, r_{12}\right), r_{2}=\phi_{\mathbf{n}}\left(r_{21}, r_{22}\right) \\ \perp & \text { otherwise }\end{cases}
$$

Important is the "otherwise" case in the second item. Here $\phi$-constants are constructed, which, as operands of ordinary operators, are evaluated in a distributive fashion (cp. lines two to four of the third item). Note, finally, that in item three the evaluation of $\mathcal{E}^{+}$yields $\perp$, if $r_{1}$ and $r_{2}$ are $\phi$-constants with different top level $\phi$-operators. This is in order to avoid the combinatoric explosion reflecting the co-NP-hardness of CP.

The analysis on the value graph is particularly easy to describe. It computes the greatest fixed point of an annotation satisfying: ${ }^{15}$

${ }^{14}$ Note that $\mathbf{D}^{\phi} \cup\{\perp, \top\}$ is not a lattice as unique greatest lower bounds do not exist due to the distinction of $\phi$-operators. For instance, $\phi_{\mathbf{n}}(2,3)$ and $\phi_{\mathbf{m}}(2,3)$ are both incomparable lower bounds of 2 and 3. However, in our application the meetoperation is always uniquely determined by the evaluation context.

15 The $\phi$-constants of the annotation can efficiently be represented using a shared representation. 
Start Annotation: For each node $n$ in the value graph initialize:

$$
\operatorname{dfi}[n]= \begin{cases}\mathcal{E}^{+}(\operatorname{lab}[n]) & \text { if } n \text { is a leaf node } \\ \top & \text { otherwise }\end{cases}
$$

Iteration Step: For a node $n$ labelled with an ordinary or $\phi$-operator $\omega$ :

$$
\operatorname{dfi}[n]=\mathcal{E}^{+}(\omega(\operatorname{dfi}[1(n)], \operatorname{dfi}[\mathrm{r}(n)])
$$

Note that the evaluation of terms (Case (1) and (3)) as well as the merge of data-flow information at join nodes (Case (2)) is both encoded in the function $\mathcal{E}^{+}$. Figure 6 provides an example that is suitable to elucidate the mechanism of the analysis.
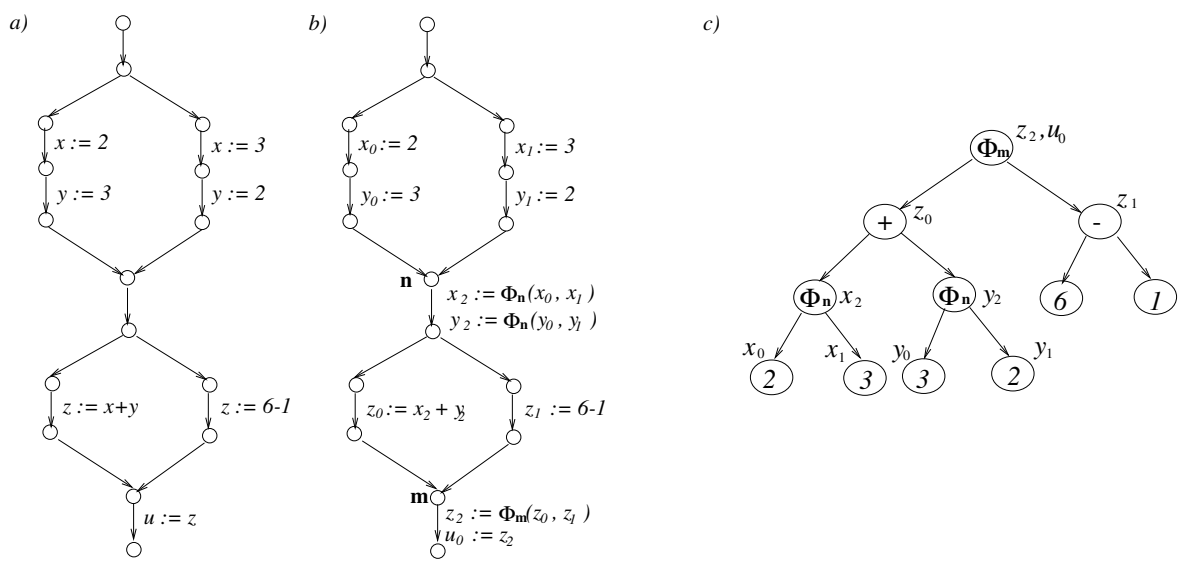

Fig. 6. (a) An example illustrating the function of our extended algorithm, (b) the program in SSA form, and (c) the corresponding value graph.

It should be noted that although $u$ is a constant of value 5 , it is neither a simple constant nor is detected by Kam and Ullman's "look-ahead-of-one" heuristics. However, our extended algorithm is capable of detecting this constant. Figure 7 shows the iteration sequence which becomes already stable after two iterations.

The most important point is the evaluation of the +-labelled node in the second iteration step which in details is due to the following sequence of transformations:

$$
\begin{aligned}
\mathcal{E}^{+}\left(+\left(\phi_{\mathbf{n}}(2,3), \phi_{\mathbf{n}}(3,2)\right)\right) & = \\
\mathcal{E}^{+}\left(\phi_{\mathbf{n}}(+(2,3),+(3,2))\right) & = \\
\mathcal{E}^{+}(+(2,3)) & =5
\end{aligned}
$$

$\left[\right.$ Since $\left.\mathcal{E}^{+}(+(2,3))=\mathcal{E}^{+}(+(3,2))\right]$

Main Results. In the following we briefly collect the most important results on the extended algorithm. First of all, it is easy to show that the extended analysis 

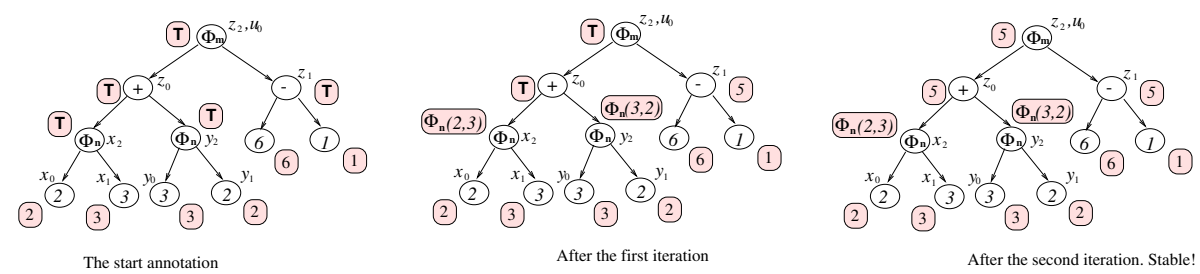

Fig. 7. Illustrating the extended analysis for Figure 6(c).

always stays above a corresponding analysis in the simple constant setting. That means that the elements of the current annotation are always greater or equal than the elements of the annotation that would be computed by a corresponding iteration strategy in the simple constant analysis. ${ }^{16}$ Hence, we particularly have:

\section{Theorem 4 (Extended Algorithm on Arbitrary Control Flow).}

On programs with arbitrary control flow the extended algorithm detects at least any simple constant.

As demonstrated in Section 5.1 detecting all constants is intractable even on acyclic programs. However, the extended algorithm is able to detect a major fragment of these constants which is characterised through the operators involved in building candidates for constant propagation. The key observation for this is that a variable which is not of a constant value at a program point $\mathbf{n}$ cannot contribute to the construction of a constant term if the operators of the term are injective in each argument and some operand variable is defined at a program point behind $\mathbf{n}$. Injectivity holds for a wide range of operators like,+- or division for real numbers but certainly not for operators like integer division used in the example of Figure 5. Multiplication is an injective operator for any argument if the respective other one can be proven different from 0 . We have:

\section{Theorem 5 (Extended Algorithm on Acyclic Control Flow).}

On programs with acyclic control flow the extended algorithm detects every constant which is composed of operators only which are injective in their relevant arguments.

In the worst case the run-time complexity of the extended algorithm is of third order in the size of the value graph. This is due to the fact that an annotation can grow up to the size of the value graph. Hence, this sets an upper bound for the number of iterations at each node where each evaluation of an annotation has costs that are linear in the size of the resulting structure. In practice, we rather expect that the annotations are reasonably small, as most of them will quickly evaluate to $\perp$.

$\overline{{ }^{16} \text { Note that } \mathbf{D}}$ is contained in $\mathbf{D}^{\phi} \cup\{\perp, \top\}$ as a sub-cpo. 


\subsection{Further Extensions: Towards Conditional Constants}

The approach we presented so far is open for extensions. Particularly interesting are extensions towards taking conditional branching into account. Wegman and Zadeck [30,31] and Click and Cooper [4] presented two completely different approaches for this. While Click and Cooper's approach proceeds by mutually feeding the results of simple constant propagation with those of a dead path analysis, Wegman and Zadeck's approach, which can be considered a specialisation of Wegbreit's general algorithm for performing global flow analysis taking conditional branches into account (cf. [29]), enhances Kildall's approach by means of "executable flags" on (flow graph) edges. In both approaches the effect is that information is not propagated along paths which are known to be infeasible. Additionally, it allows them to make use of branch conditions in specific branches, e.g., a condition like $x=1$ allows them to propagate this information along the then-branch of an if-then-else statement.

Both variants can be put on top of the CP-approach we proposed here. For example, the "executable flags" of Wegman and Zadeck can essentially be mimicked by "executable flags" on ingoing edges of $\phi$-nodes. Along the lines of Wegman and Zadeck's approach this allows the CP-algorithm on the value graph to neglect paths which are known to be not executable.

\subsection{Related Work}

Though it has certainly been considered before (cf. [5,17]), CP has particularly been pioneered by the investigations of Kildall [19], and Kam and Ullman $[15,16]$. The class of simple constants they introduced became the state-of-the-art of $\mathrm{CP}$-algorithms, though it is suboptimal even on acyclic programs. Besides a few approaches aiming at detecting more general classes of constants like those of Kam and Ullman [16], Wegman and Zadeck [30,31], and Steffen and Knoop [28], and adaptations of $\mathrm{CP}$ to advanced program settings like the interprocedural (cf. $[3,8,12,22,27,31])$ or (explicitly) parallel one (cf. $[20,21])$, there is a large group of approaches aiming at computing simple constants more efficiently.

This latter group includes the algorithms of Reif and Lewis [24,25], Ferrante and Ottenstein [9], and Wegman and Zadeck [30,31]. They are known as sparse CP-algorithms. This also applies to the algorithm of Kennedy proposed in [18]. It is even more efficient, however, less powerful as it is in contrast to the sparse "optimistic" algorithms a "pessimistic" one. In contrast to the optimistic algorithms, which essentially work by disproving constancy of a term, pessimistic ones have to explicitly prove constancy, which in the presence of loops acts like a worst-case assumption.

Common to the optimistic sparse algorithms is that they work on specific representations of the control and data flow of a program. Most similar to the value graph of Alpern, Wegman, and Zadeck [2] our algorithm operates on, are the global value graph used by Reif and Lewis [24,25], and the SSA form (cf. [7]) used by Wegman and Zadeck [30,31]. In fact, the value graph of [2] can be considered a representation, which uniformly combines the spirit underlying SSA and the global value graphs of Reif and Lewis. 


\section{Conclusions}

The value graph has been designed and used so far as a means for efficiently detecting value equivalences of program terms $[2,26]$. In this article, we demonstrated that it is not restricted to this application, but well-suited for other optimisations, too. We developed a new algorithm making constant propagation available on the value graph. Even more important, this algorithm enjoys particularly nice properties. It combines the (1) efficiency of the sparse CP-algorithms (cf. $[24,25,9,30,31])$ with the (2) conceptual simplicity of their classic conventional counterparts (cf. $[19,16]$ ), while simultaneously (3) outperforming them in power. In fact, the class of constants it detects is a proper superset of the simple constants detected by the classical algorithms, which still constitute the state-of-the-art in nowadays compilers. Moreover, the new algorithm is open for extensions. In Section 5.3 we sketched already an extension towards conditional constants along the lines of [30,31] and [4]. Other extensions we are currently investigating include extensions to variable range analysis (cf. $[13,14])$ and to interprocedural constant propagation in the fashion of $[3,31]$.

\section{References}

1. A. V. Aho, R. Sethi, and J. D. Ullman. Compilers: Principles, Techniques and Tools. Addison-Wesley, 1985. 94

2. B. Alpern, M. N. Wegman, and F. K. Zadeck. Detecting equality of variables in programs. In Conf. Rec. 15th Symp. Principles of Prog. Lang. (POPL'88), pages 1 - 11. ACM, NY, 1988. 95, 99, 107, 108

3. D. Callahan, K. D. Cooper, K. W. Kennedy, and L. M. Torczon. Interprocedural constant propagation. In Proc. ACM SIGPLAN Symp. on Compiler Construction (SCC'86), volume 21, 7 of ACM SIGPLAN Not., pages $152-161,1986$. 95, 107, 108

4. C. Click and K. D. Cooper. Combining analyses, combining optimizations. ACM Trans. Prog. Lang. Syst., 17(2):181 - 196, 1995. 107, 108

5. J. Cocke and J. T. Schwartz. Programming languages and their compilers. Courant Inst. Math. Sciences, NY, 1970. 107

6. P. Cousot and R. Cousot. Abstract interpretation: A unified lattice model for static analysis of programs by construction or approximation of fixpoints. In Conf. Rec. 4th Symp. Principles of Prog. Lang. (POPL'77), pages 238-252. ACM, NY, 1977. 98

7. R. Cytron, J. Ferrante, B. K. Rosen, M. N. Wegman, and F. K. Zadeck. Efficiently computing static single assignment form and the control dependence graph. ACM Trans. Prog. Lang. Syst., 13(4):451 - 490, 1991. 99, 107

8. E. Duesterwald, R. Gupta, and M. L. Soffa. A practical framework for demanddriven interprocedural data flow analysis. ACM Trans. Prog. Lang. Syst., 19(6):992 - 1030, 1997. 95, 107

9. J. Ferrante and K. J. Ottenstein. A program form based on data dependency in predicate regions. In Conf. Rec. 10th Symp. on Principles of Prog. Lang. (POPL'83). ACM, NY, 1983. 94, 95, 107, 108

10. M. R. Garey and D. S. Johnson. Computers and Intractability - A Guide to the Theory of NP-Completeness. W. H. Freeman \& Co, San Francisco, CA, 1979. 102 
11. S. L. Graham and M. N. Wegman. A fast and usually linear algorithm for global flow analysis. J. ACM, 23(1):172 - 202, 1976. 98

12. D. Grove and L. Torczon. Interprocedural constant propagation: A study of jump function implementation. In Proc. ACM SIGPLAN Conf. on Prog. Lang. Design and Impl. (PLDI'93), volume 28,6 of ACM SIGPLAN Not., pages 90 - 99, 1993. 95, 107

13. W. H. Harrison. Compiler analysis of the value range of variables. IEEE Trans. Softw. Eng., 3(SE-3):243 - 250, 1977. 108

14. H. Johnson. Dataflow analysis for intractable systems software. In Proc ACM SIGPLAN Symp. on Compiler Construction (SCC'86), volume 21, 7 of ACM SIGPLAN Not., pages $109-117,1986.108$

15. J. B. Kam and J. D. Ullman. Global data flow analysis and iterative algorithms. J. ACM, 23(1):158 - 171, 1976. 107

16. J. B. Kam and J. D. Ullman. Monotone data flow analysis frameworks. Acta Informatica, 7:305 - 317, 1977. 94, 95, 97, 98, 99, 101, 102, 107, 108

17. K. Kennedy. Variable subsumption with constant folding, August 1973. Courant Institute of Mathematical Sciences, New York University, SETL Newsletter 112. 107

18. K. Kennedy. A survey of data flow analysis techniques. In S. S. Muchnick and N. D. Jones, editors, Program Flow Analysis: Theory and Applications, chapter 1, pages 5 - 54. Prentice Hall, Englewood Cliffs, NJ, 1981. 94, 107

19. G. A. Kildall. A unified approach to global program optimization. In Conf. Rec. 1st Symp. Principles of Prog. Lang. (POPL'r3), pages 194-206. ACM, NY, 1973. 94, 95, 97, 98, 107, 108

20. J. Knoop. Parallel constant propagation. In Proc. 4th Europ. Conf. on Parallel Processing (Euro-Par'98), LNCS 1470, pages 445 - 455. Springer-V., 1998. 95, 107

21. Jaejin Lee, S. P. Midkiff, and D. A. Padua. A constant propagation algorithm for explicitly parallel programs. Int. J. Parallel Programming, 26(5):563 - 589, 1998. 95, 107

22. R. Metzger and S. Stroud. Interprocedural constant propagation: An empirical study. ACM LOPLAS, 2(1-4):213 - 232, 1993. 95, 107

23. S. S. Muchnick. Advanced Compiler Design and Implementation. Morgan Kaufmann, San Francisco, CA, 1997. 94, 99, 101

24. J. H. Reif and R. Lewis. Symbolic evaluation and the global value graph. In Conf. Rec. 4th Symp. Principles of Prog. Lang. (POPL'rry), pages 104-118. ACM, NY, 1977. 94, 95, 97, 107, 108

25. J. H. Reif and R. Lewis. Efficient symbolic analysis of programs. Technical Report 37-82, Aiken Computation Laboratory, Harvard University, 1982. 94, 95, 107, 108

26. O. Rüthing, J. Knoop, and B. Steffen. Detecting equalities of variables: Combining efficiency with precision. In Proc. 6th Static Analysis Symposium (SAS'99), LNCS 1694, pages 232 - 247. Springer-V., 1999. 95, 108

27. M. Sagiv, T. Reps, and S. Horwitz. Precise interprocedural dataflow analysis with applications to constant propagation. TCS, 167(1-2):131 - 170, 1996. (Special issue devoted to TOPLAS'95). 95, 107

28. B. Steffen and J. Knoop. Finite constants: Characterizations of a new decidable set of constants. TCS, 80(2):303 - 318, 1991. (Special issue devoted to MFCS'89). 94, 96, 102, 107

29. B. Wegbreit. Property extraction in well-founded property sets. IEEE Trans. Softw. Eng., 1(SE-3):270 - 285, 1975. 107 
30. M. N. Wegman and F. K. Zadeck. Constant propagation with conditional branches. In Conf. Rec. 12th Annual Symp. on Principles of Prog. Lang. (POPL'85), pages 291 - 299. ACM, New York, 1985. 94, 95, 96, 107, 108

31. M. N. Wegman and F. K. Zadeck. Constant propagation with conditional branches. ACM Trans. Prog. Lang. Syst., 13(2):181 - 210, 1991. 94, 95, 96, 101, 107, 108

32. R. Wilhelm. Global flow analysis and optimization in the MUG2 compiler generating system. In S. S. Muchnick and N. D. Jones, editors, Program Flow Analysis: Theory and Applications, chapter 5, pages 132 - 159. Prentice Hall, Englewood Cliffs, NJ, 1981. 94 